Tatiana A. Sénina (moniale Kassia)

Saint Pétersbourg

\title{
UN SAINT FOUETTE UN AUTRE : THÉOKTISTOS LE LOGOTHÈTE ET EUTHYME DE SARDES
}

Le logothète Théoktistos est connu comme un des initiateurs de la restauration de l'orthodoxie en 843 , le principal collaborateur de l'impératrice $S$. Théodora en $842-855$, et sa mémoire est célébrée par l'Église orthodoxe le 20 novembre. ${ }^{1}$ Le Recit sur la grâce à l'empereur Théophile raconte que le basileus mourant a vu sur le cou de Théoktis-

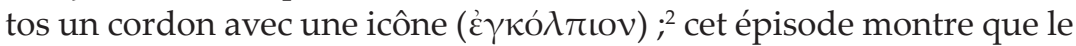
logothète serait devenu iconophile vers le début de l'année 842 . Mais l'était-il auparavant?

Pour la première fois nous rencontrons Théoktistos à la veille de la Nativité de 820 quand ce jeune eunuque, serviteur de Michel le Bègue, aide son maître à éviter la mort imminente en faisant connaître le danger du dévoilement aux amis de Michel. Ceux-ci n'ont pas tardé à assassiner l'empereur Léon l'Arménien, et après son avènement au trône Michel II a recompensé Théoktistos en lui attribuant le titre de

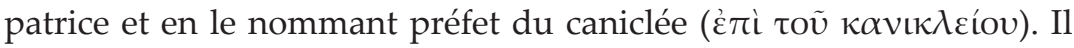
l'est resté sous Théophile et vers 842 est devenu en plus le logothète du drome ; avant sa mort l'empereur l'a nommé un des régents et tuteurs de son fils Michel III. Théoktistos a donc fait une bonne carrière sous les empereurs iconoclastes, et les derniers ordres de Théophile indiquent que l'empereur ne soupçonnait point Théoktistos de sympathie envers les iconophiles. Le Continuateur de Théophane dit que Théophile, inquieté par une prédiction du rétablissement de la vénération

(1) R.-J. Lilie, C. Ludwig, Th. Pratsch, I. Rochow (Hrsg.), Prosopographie der Mittelbyzantinischen Zeit. Erste Abteilung (641-867) (Berlin, 1998) \# 8050 ; la date de la mémoire y est indiquée de manière érronée le 20 octobre, cf. C. De Smedt, I. De Backer, F. Van Ortroy, I. Van den Gheym, H. Delehaye, A. Poncelet (eds.), Propylaeum ad Acta Sanctorum Novembris. Synaxarium Ecclesiae Constantinopolitanae (Bruxellis, MDCCCCII; CD-éd.: Cambridge, 2002) 244.16-18.

(2) BHG 1734, l'édition critique avec la traduction russe cf. Д. Е. АФИногенов, «Повесть о прощении императора Феофила» и Торжество Православия (Москва, 2004) (Scrinium Philocalicum, IV) 96.131sq. 
des icônes après sa mort, a fait promettre sa femme et Théoktistos de ne pas détrôner le patriarche Jean le Grammairien ni de revenir au culte des images. ${ }^{3}$ Assurément Théoktistos le lui avait promis, autrement il n'aurait pas pu être nommé régent, - mais on sait qu'il n'a point tenu sa promesse, et que le conseil qui a condamné Jean a eu

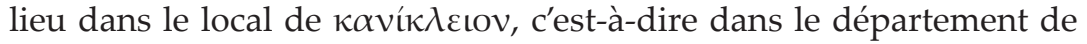
ce même Théoktistos. ${ }^{4}$

On peut apprendre davantage sur ce fonctionnaire en lisant la Vie de S. Euthyme de Sardes, confesseur iconophile martyrisé en 831. S. Méthode, l'auteur de cette Vie et témoin des derniers jours du confesseur, écrit que trois fonctionnaires ayant interrogé et flagellé $S$. Euthyme le 18 décembre 831 étaient le logothète, le préposé du caniclée et un manglavite. ${ }^{5} J^{\prime}$ ai déjà démontré que le logothète du drome mentionné dans la Vie était Arsabère, le mari de Kalomarie, sœur de l'impératrice Théodora. ${ }^{6}$ Mais le préposé du caniclée ne pouvait être nul autre que Théoktistos. En effet, il occupait ce poste sous Michel II, donc avant 831, et il

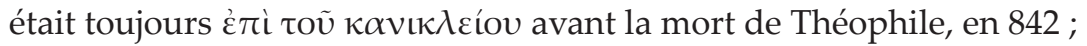
nous n'avons aucune raison de penser que Théoktistos avait pu quitter sa fonction entre ces dates. ${ }^{7}$ Ainsi, au début du règne de Théophile ce futur partisan de la restauration de l'orthodoxie était non seulement un iconoclaste convaincu, mais l'un de ces « misérables » ${ }^{8}$ qui sont arrivés à l'îlot Saint-André pour interroger et fouetter le confesseur orthodoxe S. Euthyme et, au fond, l'ont fait mourir sous le fouet. ${ }^{9}$

(3) Theophanes Continuatus, Chronographia, dans : I. Bekкer (ed.), Theophanes Continuatus, Ioannes Cameniata, Symeon Magister, Georgius Monachus (Bonn, 1838) (CFHB) 121.21-122.5.

(4) Cf. Афиногенов, «Повесть о прощении илператора Феофила»..., 63.

(5) J. Gouillard, La Vie d'Euthyme de Sardes († 831), une œuvre du patriarche Méthode, Travaux et Mémoires 10 (1987) 45, § 18.339-341.

(6) Et qu'il n'est pas identique au frère de Jéan le Grammairien ; cf. Т. А. СенинА (монахиня Кассия), Несколько замечаний по поводу Жития св. Евфимия Сардского, Scr 2 (2006) 408-411.

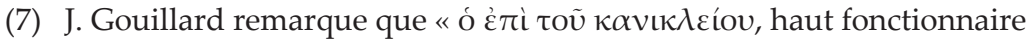
de la chancellerie impériale, apparaît précisément au début du IX ${ }^{\mathrm{e}} \mathrm{s}$., avec Théoktistos, puis Bardas ", mais, on ne sait pourquoi, il n'a pas identifié franchement le préposé de la Vie avec Théoktistos (Gouillard, La Vie d'Euthyme de Sardes..., 45, n. 97 ; cf. aussi p. 9-10).

(8) Gouillard, La Vie d'Euthyme de Sardes..., 47, § 19.363.

(9) Le saint est mort huit jours après la flagellation. Le troisième de ses tourmenteurs, le manglavite, devait s'appeller Kosmas ; cf. ibid., 10 ; СенинА, Несколько замечаний..., 408. 
S. Méthode dans la Vie du confesseur reproduit un dialogue intéressant entre $\mathrm{S}$. Euthyme et le préposé du caniclée. Malgré la flagellation cruelle le saint, ayant reçu 130 coups de fouet, refuse de nommer ceux qu'il a visité pendant son séjour à Constantinople.

«Alors ... le préposé au kaniklion ... : “Ne t'imagine pas que tu en resteras là et que tu couperas court au interrogatoires; sache que nous sommes venus pour te châtier." Le saint lui répondit : “On m’a dit que tu as ta mère et une sœur sous l'habit monastique." Et lui, sèchement : "Oui, dit-il”; ; et le saint : "Les beaux traits, le beau modèle de piété que tu portes en toi!” Lui, comme foudroyé par l'ironie du bienheureux et ayant avalé le mot comme une pierre qui lui fût restée en travers du gosier, fit taire sa méchanceté et descendit avec les autres pour se retirer. ${ }^{10}$

Ainsi, les paroles de S. Euthyme ont fait honte à Théoktistos, mais ne l'ont pas fait revenir à l'orthodoxie: nous avons vu qu'il n'a pas donné de soupçons à Théophile jusqu'à la mort de celui-ci, bien qu'il ait pu commencer à vénérer les icônes en secret - au moins l'épisode avec son $\dot{\varepsilon} \gamma \kappa o ́ \lambda \pi \iota$ ov peut l'indiquer.

Il est assez intéressant de savoir quand et par qui la mémoire de Théoktistos a été inclue dans le Synaxaire. Il est peu probable que cela ait eu lieu pendant le patriarcat de S. Photios : le protoasécrite sous Théodora et, à cause de sa fonction, le collaborateur le plus proche du préfet du caniclée, Photios n'a point protesté contre l'assassinat de Théoktistos en 855. Après l'expulsion de Théodora et l'arrivée au pouvoir de Michel III avec le césar Bardas, Photios est remplacé sur le patriarcat par S. Ignace, créature de Théodora et Théoktistos; et Bardas, le principal complice du meurtre de Théoktistos, est resté jusqu'à sa mort un grand ami du nouveau patriarche. On peut donc supposer que l'insertion de Théoktistos dans le Synaxaire en tant que martyr a eu lieu pendant le deuxième patriarcat d'Ignace, entre 867 et 877 .

(10) § 19, je cite la traduction de Goulllakd, La Vie d'Euthyme de Sardes..., 48 . 